\title{
Displacement Measurement and Study of Surface Roughness using Laser Speckle Technique
}

\author{
R.Balamurugan $^{\mathrm{a}} *$, S.Muruganand ${ }^{\mathrm{b}}$ \\ ${ }^{a}$ Dept.of Physics, Kumaraguru College of Technology, Coimbatore - 641 049, India. \\ ${ }^{b}$ Dept.of Electronics \& Instrumentation, Bharathiyar University, Coimbatore -641046, India. \\ *Corresponding Author: balamurugan.r.sci@kct.ac.in
}

\begin{abstract}
Laser Speckle images have been taken before and after the displacement were made. Subtraction of the speckle patterns gives the displacement. Binary speckles are used to study surface roughness and compared with stylus profilometer method.
\end{abstract}

Keywords: Laser speckle, Displacement, Surface roughness, Binary speckle, Stylus profilometer.

\section{Introduction}

In industry there are now strong demands for non contacting and quick measurement of surface shape and deformation of mechanical parts and products of various materials and shapes. The conventional tactile instruments cannot be used for those of soft materials and/or complex shape. Scanning of mechanical probes also takes much time and is not suited for quick and in-process measurement. Optical methods, especially interferometry techniques, which are free from these issues, have been mainly applied to optically smooth surfaces. Surface shape measurement on rough objects has also been based on geometrical optics such as triangulation and grating projection [1].

\section{Displacement Measurement}

When an optically rough surface is illuminated with a coherent beam, a high contrast granular structure, known as speckle pattern is formed in the space is known as objective speckle pattern. It can also be observed at the image plane of a lens and it is then referred as subjective speckle pattern. The scattering regions are statistically independent and uniformly distributed between $-\pi$ and $\pi$. [2]. The speckles in the pattern undergo both positional and intensity changes when the object is deformed.
Illumination of a rough surface with coherent light produces a random intensity distribution in front of the surface, called speckle pattern [3]. Because the speckle pattern follows the movement of the scattering surface the speckle can be used for displacement/deformation measurement [4].

The optical setup for speckle interferometry is based on the Michelson interferometer [5]. The phase change of each speckle before and after deformation is recorded and it depends upon the change in optical path length between the exposures and contains the displacement information.

$$
I_{\text {before }}=I_{o b j}+I_{r e f}+2 \sqrt{I_{o b j} I_{r e f}} \cos \left(\varphi_{0}\right)
$$

Where, $I_{o b j}$ and $I_{\text {ref }}$ are the intensities of the object and reference beams respectively. $\varphi_{0}$ is the unknown and random initial phase distribution of the speckle pattern at that point.

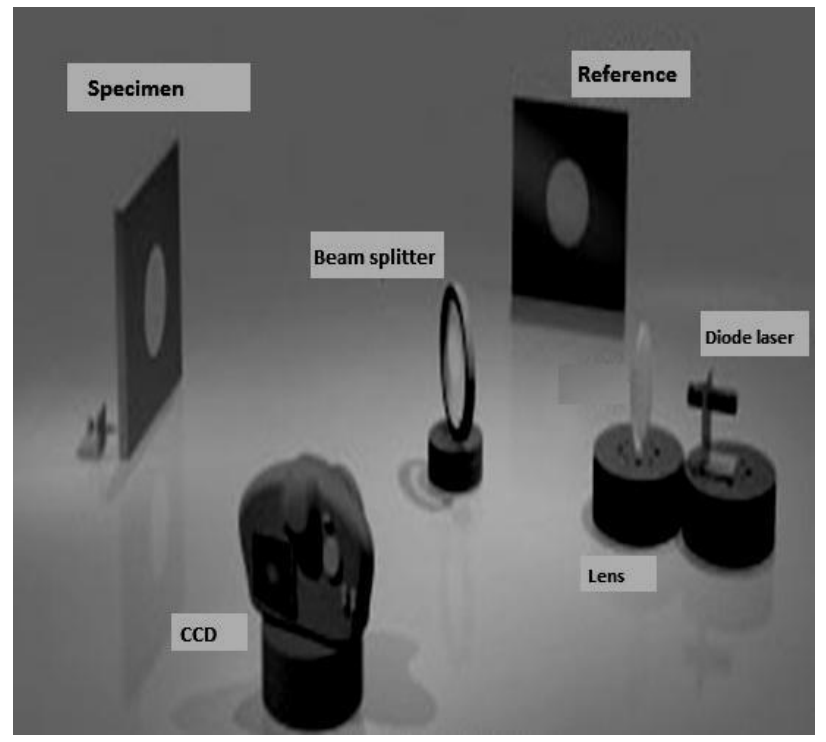

Fig.1 Schematic Michelson Interferometer setup 
When the object moves, the phase distribution undergoes a change and the intensity at the object is:

$$
I_{a f t e r}=I_{o b j}+I_{r e f}+2 \sqrt{I_{o b j} I_{r e f}} \cos \left(\varphi_{0}+\varphi_{o b j}\right)
$$

where, $\Delta \varphi$ obj is the phase change induced by object displacement. The difference between $\mathrm{I}_{\text {before }}$ and $\mathrm{I}_{\text {after }}$ calculation is a simple approach to measure $\Delta \varphi_{\text {obj}}$.

$$
I=I_{\text {after }}-I_{\text {before }}=2 \sqrt{I_{o b j} I_{r e f}}\left[\cos \left(\varphi_{0}+\Delta \varphi_{o b j}\right)-\cos \varphi_{0}\right]
$$

which can be rewritten as:

$\Delta I=4 \sqrt{I_{o b j}} \sqrt{I_{r e f}} \sin \left\{\varphi_{0}+\frac{\Delta \varphi_{o b j}}{2}\right\} \sin \Delta \varphi_{o b j / 2}$

The intensity change $\Delta \mathrm{I}$ depends on the random initial phase distribution $\varphi_{0}$ as well as on the deformation induced phase change $\Delta \varphi_{\mathrm{obj}}$. The phase jump of $2 \pi$ shows that the line of constant displacement.

\section{Results of Displacement measurement}

The various positions of speckle images are shown below:

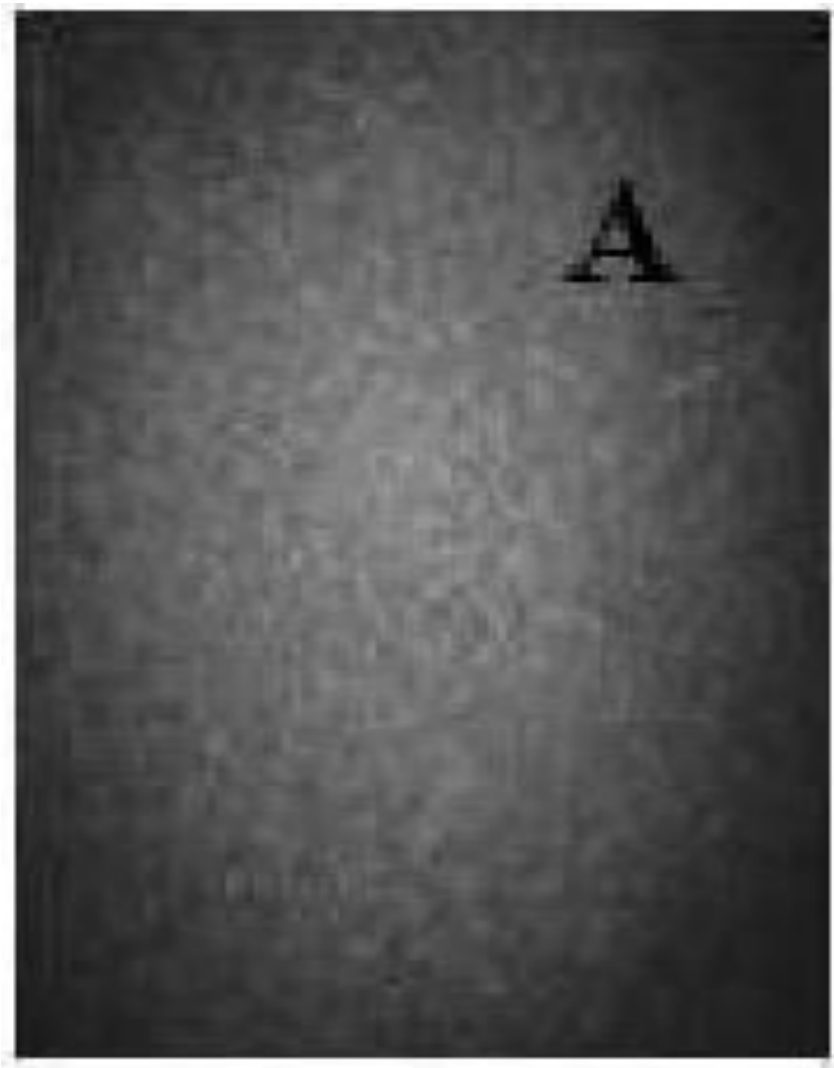

Fig. 2 (a) Speckle image-Before displacement

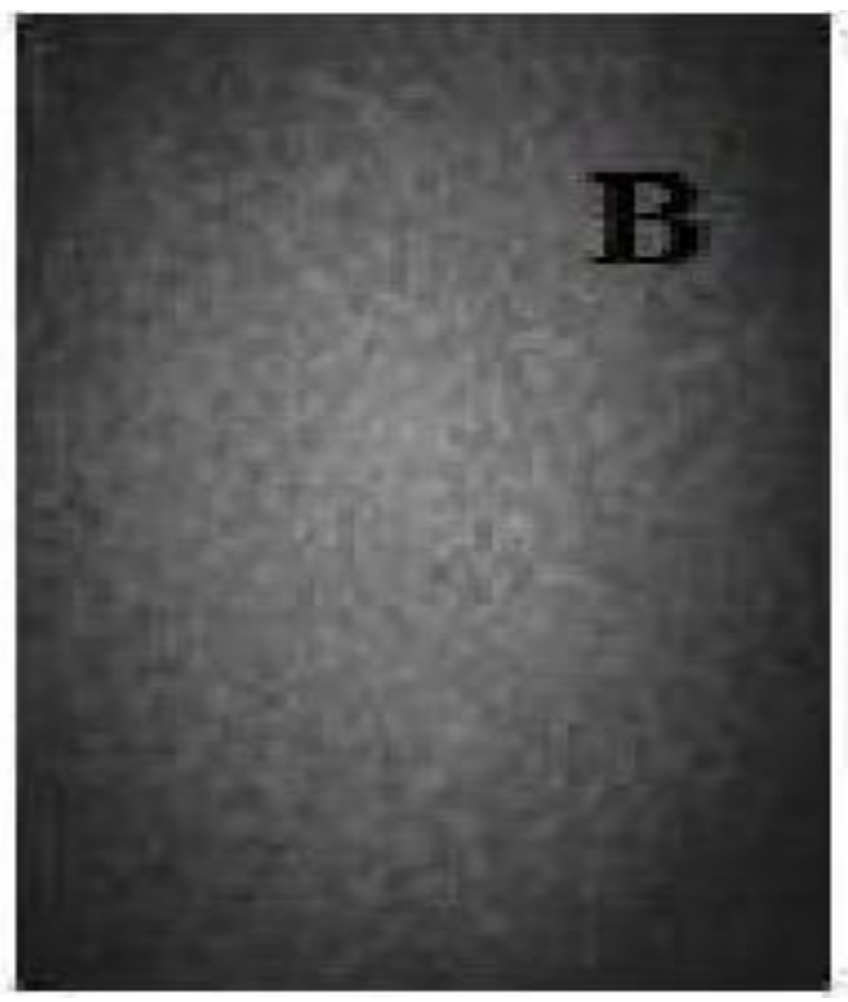

Fig.2 (b) Speckle image-After displacement

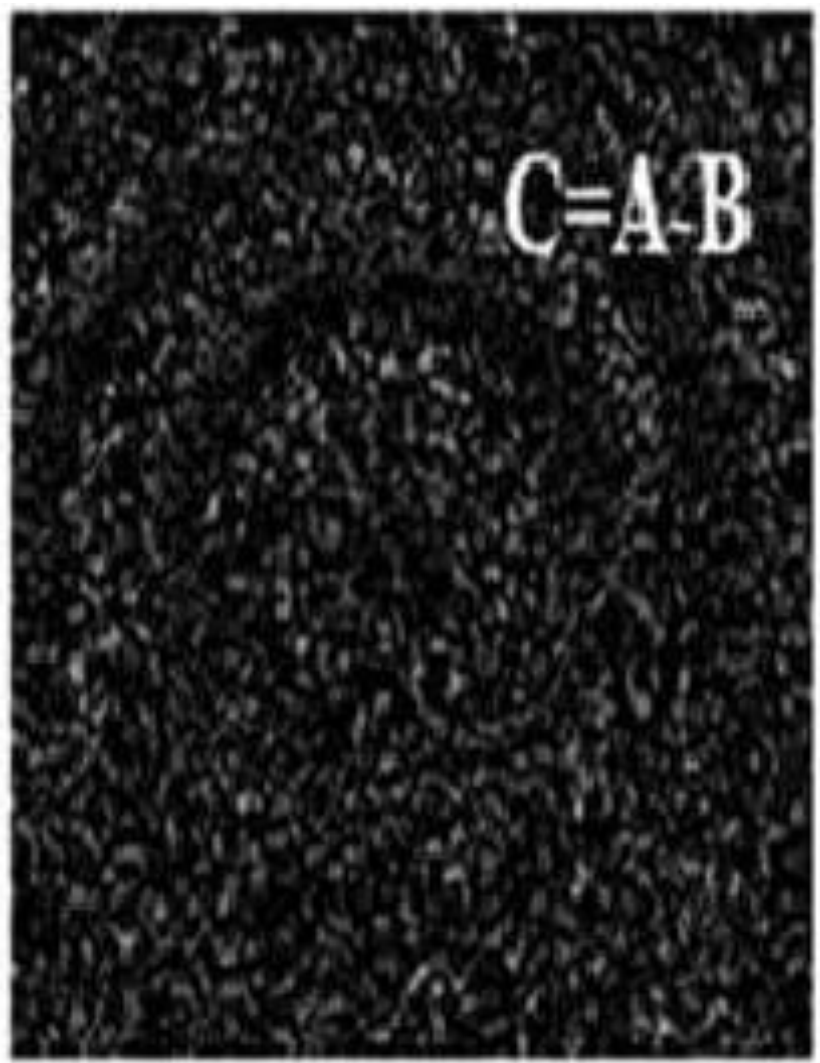

Fig.2 (c) Specklegram: Subtracted image. 


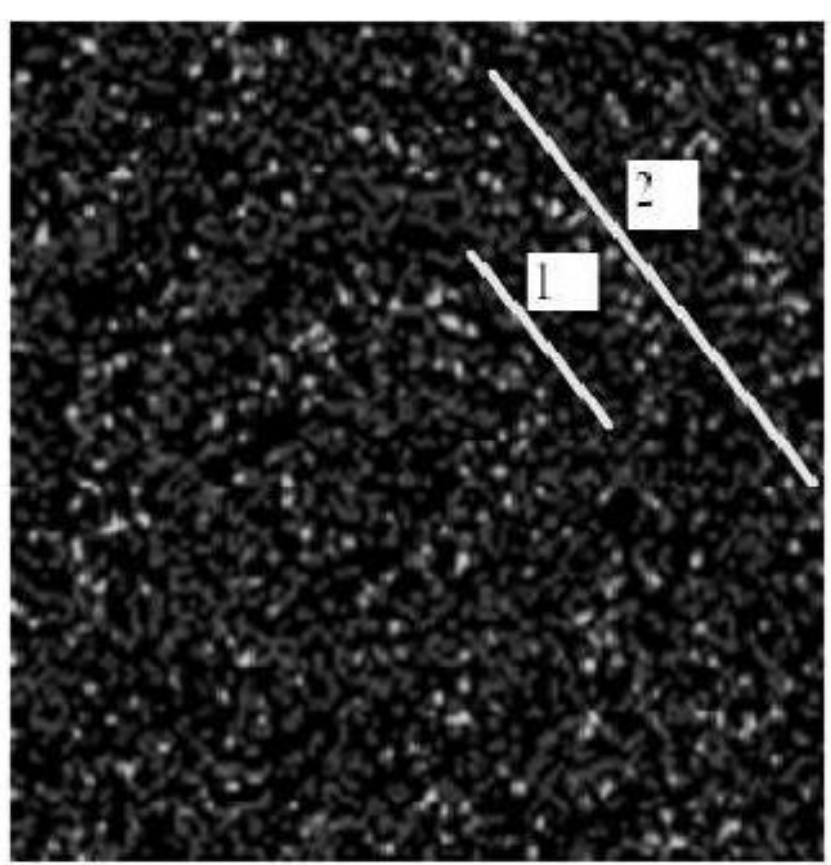

Fig.3 (a) Fringe counting

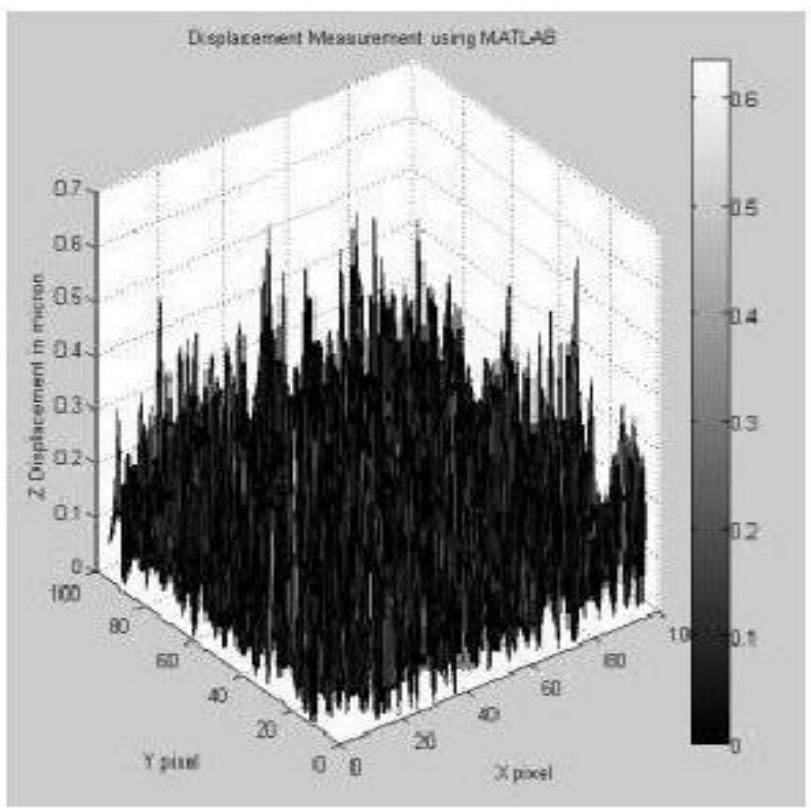

Fig.3 (b) Displacement Peak in micron

\section{Surface Roughness}

Surface roughness can be regarded as the variation of surface height. The most obvious parameter is the average departure of surface height from its mean value, usually called the average roughness or the center line average roughness. This is the parameter most frequently used for machined surfaces and is usually designated by the symbol $\mathrm{R}_{\mathrm{a}}$.
When a light beam is reflected by a rough surface it is well known that the intensity of the speckle pattern depends on the surface roughness. Milled samples were illuminated using a coherent light source a $\mathrm{CW} \mathrm{He}-\mathrm{Ne}$ laser with normal incidence.The scattered light at the specular direction was recorded at the image plane by means of a CCD camera associated with a long distance microscope.

\section{B/D Counting \& Stylus profile graph}

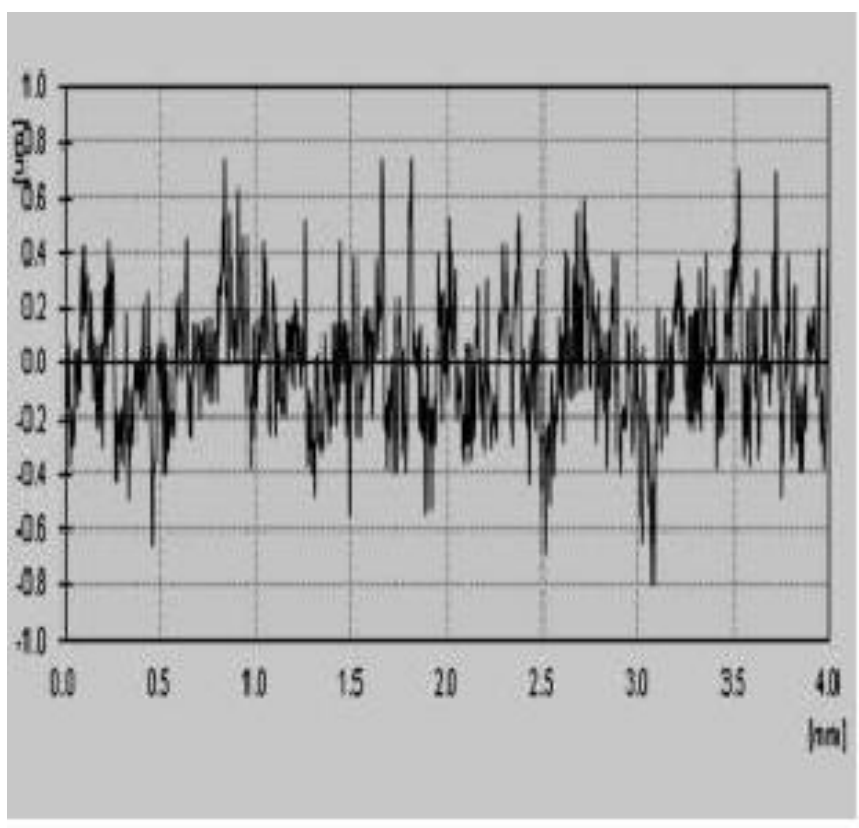

Fig.5(a) Stylus Profile graph for speckle image-1

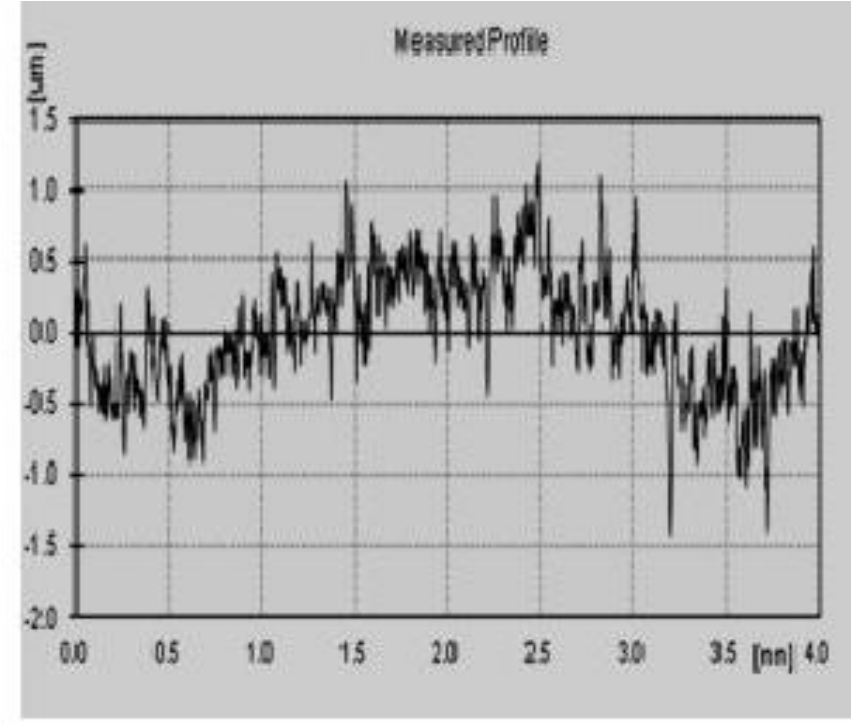

Fig.5(b) Stylus Profile graph for speckle image-2 


\section{B/D Count Method:}

The number of bright (B) and dark (D) pixels and the ratio $B / D$ are related with the effects of the surface roughness on the speckle pattern. With the digital image of speckle patterns, the calculation of $\mathrm{B} / \mathrm{D}$ ratio is performed using an image processing algorithm implemented in MATLAB. Images in grayscale should be converted in a binary image. For each pixel of this image a pixel value has been assigned within grayscale.

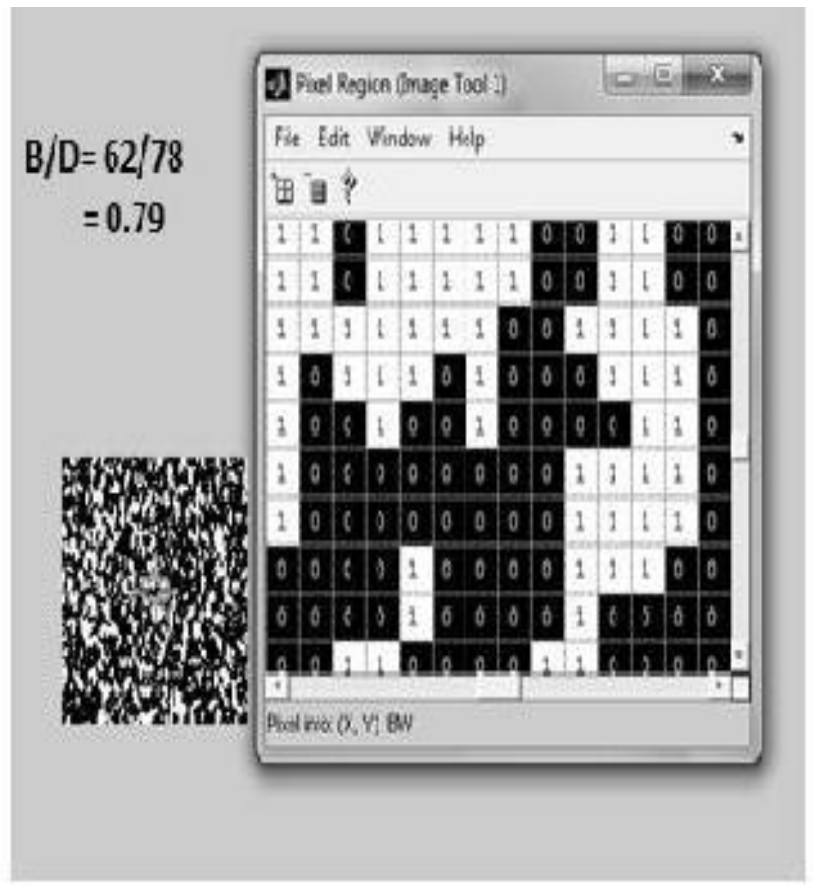

Fig.4 (a) B/D Counting for speckle image-1

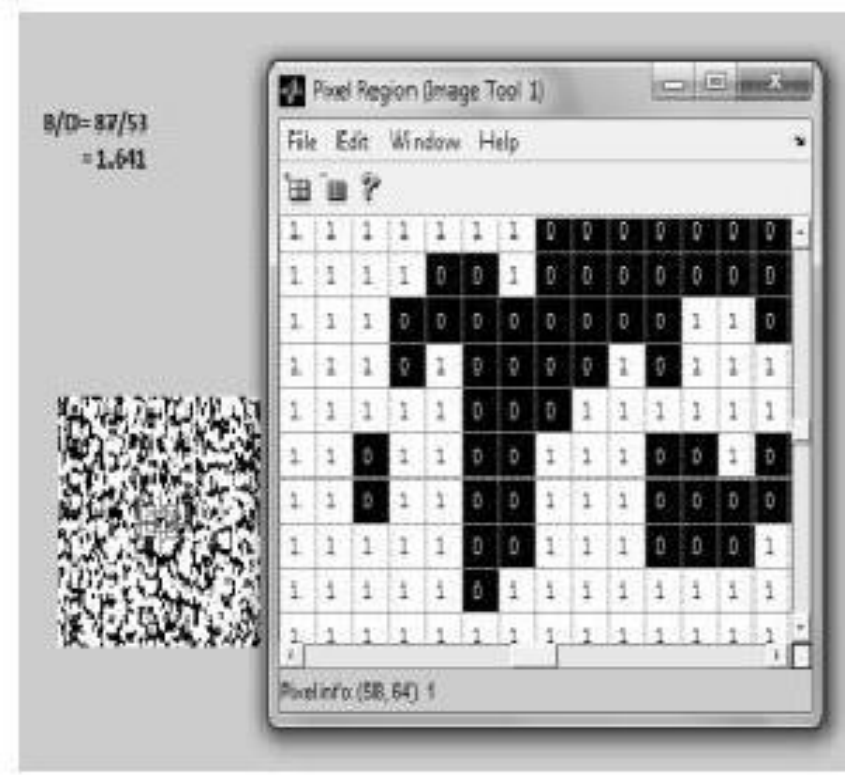

Fig.4 (b) B/D Counting for speckle image-2

\section{Conclusions}

In this paper, applications of speckle patterns for the measurements of displacement and surface roughness have been described. The peak shows the displacement value is 0.6 micron which satisfied with conventional gauge method. The B/D ratio results are in good agreement with the stylus profilometer measured values. It is found that the ratio between bright and dark regions of the binarized speckle patterns exponentially increases when the surface roughness increases.

\section{Acknowledgment}

The author very sincerely thanks to the management of Kumaraguru College of technology, especially the Chairman Dr.N.Mahalingam for encouraging and supporting R\&D work in the organization.

\section{References}

(1) Ichirou Yamaguchi "Correlation Techniques in Speckle Metrology and Digital Holography”, Proc. of SPIE Vol. 7008, 70081E, (2008), pp E1-E10.

(2) J.W.Goodman, "Some fundamental properties of speckle", J opt.Soc.Am, 1145-1149, (1966).

(3) Jones R Wykes C, "Holography and speckle interferometry", 2nd ed, Cambridge University Press, New York, USA, 122-142,(1989).

(4) R. S. Sirohi "Speckle Metrology", Marcel Dekker, New York, (1993).

(5) R.K. Erf "Speckle Metrology", Academic Press, New York, (1978).

(6) J. C. Dainty "Laser Speckle and Related Phenomena", Springer- Verlag, Berlin, 2nd ed. (1984). 